\title{
Hemorrhagic risk after intravenous thrombolysis for ischemic stroke in patients with cerebral microbleeds and white matter disease
}

\author{
Maria Luisa Capuana ${ }^{1} \cdot$ Svetlana Lorenzano ${ }^{2} \cdot$ Maria Chiara Caselli $^{3} \cdot$ Maurizio Paciaroni $^{4} \cdot{\text { Danilo } \text { Toni }^{2}}^{2}$
}

Received: 6 July 2020 / Accepted: 12 September 2020 / Published online: 29 September 2020

(C) The Author(s) 2020

\begin{abstract}
Objectives Aim of this study was to evaluate the association between cerebral microbleeds (CMBs) and white matter disease (WMD) with intracerebral hemorrhage (ICH) after intravenous thrombolysis (IVT) with rt-PA. We also evaluated whether CMBs characteristics and WMD burden correlate with symptomatic ICH and outcome.

Methods We included acute ischemic stroke (AIS) patients treated with IVT. The number and location of CMBs as well as severity of WMD were rated analyzing pre- or post-treatment MRI. Multivariable regression analysis was used to determine the impact of CMB and WMD on ICH subgroups and outcome measures.

Results 434 patients were included. CMBs were detected in $23.3 \%$ of them. ICH occurred in $34.7 \%$ of patients with CMBs. Independent predictors of parenchymal hemorrhage were the presence of CMBs (OR 2.724, 95\% CI 1.360-5.464, $p=0.005)$ as well as cortical-subcortical stroke (OR 3.629, 95\% CI 1.841-7.151, $p<0.001)$ and atherothrombotic stroke subtype (OR 3.381, 95\% CI 1.335-8.566, $p=0.010$ ). Either the presence, or number, and location of CMBs, as well as WMD, was not independently associated with the development of SICH. No independent association between the presence, number, or location of CMBs or WMD and outcome measures was observed.

Conclusions The results of our study suggest that the exclusion of eligible candidates to administration of IV rt-PA only on the basis of CMBs presence is not justified. The clinical decision should be weighed with a case-by-case approach. Additional data are needed to evaluate the benefit-risk profile of rt-PA in patients carrying numerous microbleeds.
\end{abstract}

Keywords Cerebral microbleeds $\cdot$ Intravenous thrombolysis $\cdot$ Intracerebral hemorrhage $\cdot$ White matter disease $\cdot$ Outcome

Electronic supplementary material The online version of this article (https://doi.org/10.1007/s10072-020-04720-y) contains supplementary material, which is available to authorized users.

\section{Danilo Toni}

danilo.toni@uniroma1.it

1 IRCCS Centro Neurolesi "Bonino Pulejo", Palermo, Italy

2 Emergency Department Stroke Unit, Department of Human Neurosciences, Policlinico Umberto I Hospital, Sapienza University of Rome, Viale del Policlinico, 155, 00161 Rome, Italy

3 Department of Clinical and Sperimental Medicine, University of Pisa, Pisa, Italy

4 Stroke Unit and Division of Internal and Cardiovascular Medicine, Santa Maria della Misericordia Hospital, University of Perugia, Perugia, Italy

\section{Background}

Cerebral microbleeds (CMBs) are the expression of cerebral small vessel disease (SVD) and are commonly found in the elderly population, including patients with acute ischemic stroke (AIS) undergoing intravenous thrombolysis (IVT) [1]. Microbleeds can be discriminated from macrobleeds with specific sequences of magnetic resonance imaging (MRI) by various size cut-points. Typically, CMBs have a maximum diameter of $10 \mathrm{~mm}$ and appear larger on GRE sequences compared with the other tissue lesions because of the described "blooming effect" of the MR signal at the border of these lesions [2].

If CMBs represent a risk for the development of symptomatic intracerebral hemorrhage (SICH), or predict worse outcome after IVT in AIS, is a matter of debate. Another neuroimaging marker of SVD that could be in relation to the risk of ICH is white matter disease (WMD). Some evidence suggests that it can increase the risk of ICH during IVT but, so far, it 
does not represent an absolute exclusion criterion [3]. No clear leukoaraiosis volume threshold has been identified below in which no benefit or harm of IVT could be observed [4].

\section{Aims}

The aim of this study was to evaluate the relationship between the presence and burden of CMBs and WMD with the development of ICH and SICH, and with clinical outcome after IVT in patients with AIS.

\section{Methods}

\section{Study population and data collection}

Patients with AIS receiving IVT according to the current guidelines [5] and undergoing magnetic resonance imaging (MRI) before or within $24 \mathrm{~h}$ after rt-PA infusion in two stroke centers were included in the study. We excluded patients for whom image quality was poor due to motion artifacts and patients treated with endovascular revascularization.

We collected demographic data (age, sex), baseline NIHSS score, systolic blood pressure and serum glucose levels, risk factors for stroke, pre-stroke therapies, etiopathogenetic subtypes, and neuroimaging variables.

\section{MRI protocol}

Patients included in this study underwent cranial 1.5-T MRI. The imaging protocol consisted of T1-weighted imaging, T2weighted imaging, T2*-weighted GRE imaging, fluidattenuated inversion recovery (FLAIR), diffusion-weighted imaging (DWI), perfusion-weighted imaging (PWI), apparent diffusion coefficient (ADC), and 3-dimensional time-of-flight magnetic resonance angiography. We evaluated stroke characteristics, including vascular territory, and anatomical location. Micro-hemorrhages were identified according to a field guide of CMBs detection and interpretation [2]. CMB mimics were excluded. We recorded $\mathrm{CMBs}$ number, size $(\mathrm{CMB}<5$ $\mathrm{mm}, 5 \mathrm{~mm}>\mathrm{CMB}<10 \mathrm{~mm}$ ), and their location: (1) lobar, if present in the cortex, subcortex, and white matter of the frontal, parietal, temporal, occipital, and insular lobes; (2) deep and infratentorial, if present in the head of the caudate, putamen, globus pallidus, internal capsule, thalamus, midbrain, pons, medulla, and cerebellum, according to The Brain Observer MicroBleeds Scale (BOMBS) [6]. WMD was defined as a hyperintense lesion on FLAIR and T2-weighted imaging, which was usually not seen or showed faint hypointensity on T1-weighted imaging. We rated WMD in the hemisphere contralateral to the index stroke using the scoring system presented by Fazekas et al. [7].

\section{Outcome measures}

ICH was classified by using clinical and radiological criteria as follows: hemorrhagic infarction (HI, including HI-1 and $\mathrm{HI}-2)$ and parenchymal hemorrhage ( $\mathrm{PH}$, including $\mathrm{PH}-1$ and $\mathrm{PH}-2)$, parenchymal remote hemorrhage (PHr1, PHr2) [8]. An ICH was defined as symptomatic ( $\mathrm{SICH})$ according to the SITS-MOST criteria in case of a local or remote $\mathrm{PH}-2$ on the 22 to $36 \mathrm{~h}$ post-treatment imaging scan, combined with a neurological deterioration $\geq 4$ points on the NIHSS score from baseline, or from the lowest NIHSS value between baseline and $24 \mathrm{~h}$, or leading to death. [9]. Definitions of SICH according to NINDS (the National Institutes of Neurological Disorders and Stroke) [10] and ECASS (the European Cooperative Acute Stroke Study) [8] criteria were also used. Additional outcome measures were mortality at 7 days and mortality and functional dependency at 3 months. The latter was assessed by trained personnel using the modified Rankin Scale (mRS) score, by in-person or telephone interview [11], and was used as a dichotomous variable with mRS 3-6 defined as an unfavorable functional outcome.

\section{Statistical methods}

We performed descriptive and univariate analyses for baseline clinical, demographic, and neuroimaging data, as well as outcome measures, comparing patients with and without CMBs. Percentages were calculated excluding missing cases. Continuous variables were expressed as mean (standard deviation, SD) or median (interquartile range, IQR) values based on their normal or non-normal distribution. Dichotomous variables between groups were compared by using chi-square or Fisher exact test, while Student's $t$ test or Mann-Whitney $U$ test was used for continuous variables, depending on the normality of their distribution. Variables with univariate $p$ value $<$ 0.05 were included in the multivariable models. Multivariable logistic regression analyses were performed to identify variables independently associated with the risk of developing HI or $\mathrm{PH}$ and to evaluate whether CMBs and WMD were predictors of 90-day functional outcome and mortality at 7 days and 3 months. Statistical significance was set at a probability value of $<0.05$. Data were analyzed using SPSS statistical software (IBM Corp, SPSS Statistics for Windows, Version 24, Armonk, NY).

\section{Results}

In this study, we included 434 AIS patients (60.8\% men; mean [SD] age 68.3 [13.5]) treated with IVT rt-PA $0.9 \mathrm{mg} / \mathrm{kg}$ and undergoing brain MRI over a period of approximately 5 years in two experienced stroke centers. We excluded 68 patients from the analysis because, given the presence of 
contraindications or particular clinical severity, they were unable to undergo MRI, they had poor MR image quality due to motion artifacts, or they received endovascular treatment.

Overall, the majority of patients (approximately $81 \%$ ) underwent MRI after IVT within $24 \mathrm{~h}$ of stroke onset, while only in $19 \%$ of patients MRI was performed before IVT. The median time from rt-PA bolus to MRI was 1140 (IQR 3841842) $\min$ (i.e., $19 \mathrm{~h}$ [IQR 6.4-30.7]) (Table 1). Demographics and baseline clinical and radiological characteristics of the whole cohort of patients and of the two subgroups defined by the presence/absence of CMBs are shown in Tables 1 and 2. Overall, CMBs were detected in 101 of 434 patients $(23.3 \%)$. In these subjects, the mean (SD) number of CMBs was 2.45 (3.61), with a range from 1 to 27; the majority of patients had from 1 (59.4\%) to 4 (30.7\%) CMBs. CMBs were lobar in $73.3 \%$ of cases, deep/infratentorial in $41.6 \%$ of patients, and both lobar and deep/infratentorial in $14.9 \%$ of cases (Table 2). At univariate analysis, patients with CMBs were more likely to have a history of hypertension compared to those without $(76.2 \%$ vs $62.5 \%, p=0.011)$ (Table 1 ) and had more frequently a moderate to severe WMD (Fazekas Rating Scale score 2: $31.7 \%$ vs $16.5 \%, p=0.001$; Fazekas Rating Scale score 3: $13.9 \%$ vs 3.0\%, $p<0.001$ ); WMD was absent in a higher proportion of patients without CMBs (36.6\% vs $17.8 \%, p<0.001)$ (Table 2 ). The proportion of patients with a cortical-subcortical index stroke was $44.3 \%$ in patients with CMBs and $32.4 \%$ in those without CMB ( $p$ $=0.031)$ (Table 2).

Overall, ICH (all types) were detected in 107 (24.8\%) patients of the whole cohort, and more frequently in patients

Table 1 Baseline clinical characteristics of the whole cohort of patients and of the two subgroups identified by the presence/absence of CMBs

\begin{tabular}{|c|c|c|c|c|}
\hline & All $(n=434)$ & CMBs $(n=101)$ & No CMBs $(n=333)$ & $p$ value \\
\hline Age $($ mean \pm SD) & $68.3 \pm 13.5$ & $69.0(12.6)$ & $68.1(13.8)$ & 0.517 \\
\hline Sex (males) $(\%)$ & $264(60.8)$ & $66 / 101(65.3)$ & $198 / 333(59.5)$ & 0.288 \\
\hline \multicolumn{5}{|l|}{ Vascular risk factors } \\
\hline Hypertension (\%) & $285 / 434(65.7)$ & $77 / 101(76.2)$ & $208 / 333(62.5)$ & 0.011 \\
\hline Diabetes mellitus (\%) & $74 / 434(17.1)$ & 15/101 (14.9) & $59 / 333(17.7)$ & 0.502 \\
\hline Atrial fibrillation (\%) & $70 / 433(16.2)$ & 17/101 (16.8) & $53 / 332(16.0)$ & 0.836 \\
\hline Current smoking (\%) & $82 / 429(19.1)$ & $18 / 100(18.0)$ & $64 / 329(19.5)$ & 0.746 \\
\hline Previous smoking (\%) & $64 / 419(15.3)$ & $16 / 97(16.5)$ & 48/322 (14.9) & 0.703 \\
\hline Hypercholesterolemia (\%) & $138 / 434(31.8)$ & $30 / 101(29.7)$ & $108 / 333(32.4)$ & 0.606 \\
\hline Previous stroke within 3 months (\%) & $3 / 434(0.7)$ & $1 / 101(1.0)$ & $2 / 333(0.6)$ & 0.549 \\
\hline Previous stroke earlier than 3 months (\%) & $43 / 434(9.9)$ & 9/101 (8.9) & $34 / 333(10.2)$ & 0.702 \\
\hline Previous diagnosis of TIA/amaurosis (\%) & $14 / 434(3.2)$ & $3 / 101(3.0)$ & $11 / 333(3.3)$ & 1.0 \\
\hline Congestive heart failure $(\%)$ & $10 / 432(2.3)$ & $2 / 100(2.0)$ & $8 / 332(2.4)$ & 1.0 \\
\hline \multicolumn{5}{|l|}{ Medication history } \\
\hline Prior antiplatelet therapy $(\%)$ & $137 / 434(31.6)$ & $33 / 101(32.7)$ & $104 / 333(31.2)$ & 0.785 \\
\hline Prior oral anticoagulation (\%) & $16 / 415(3.9)$ & $7 / 98(7.1)$ & $9 / 317(2.8)$ & 0.069 \\
\hline Baseline NIHSS score, median (IQR) & $8(5-15)$ & $9(5-15)$ & $8(5-14)$ & 0.682 \\
\hline Baseline systolic blood pressure $(\mathrm{mm} / \mathrm{hg})(\mathrm{mean} \pm \mathrm{SD})$ & $146.8 \pm 23.5$ & $149.0(24.9)$ & $146.1(23.1)$ & 0.285 \\
\hline Baseline diastolic blood pressure $(\mathrm{mmHg})(\mathrm{mean} \pm \mathrm{SD})$ & $80.7(13.8)$ & $80.4(14.6)$ & $80.8(13.5)$ & 0.800 \\
\hline Baseline glucose level (mg/dl), median (IQR) & $120.5(104-149.25)$ & $121.5(107.25-150)$ & $120.50(104-147.25)$ & 0.821 \\
\hline Onset-to-treatment time, median (IQR) & $180(135.0-222.25)$ & $187.50(145-229.25)$ & $180(130-220)$ & 0.121 \\
\hline Door-to-needle time, median (IQR) & $80.50(55.0-116.25)$ & $84(55-110)$ & $79(53-118)$ & 0.675 \\
\hline Bolus-MRI time, median (IQR) & $1140(384-1842)$ & $1116(363.50-2169.50)$ & $1179(388.50-1769.25)$ & 0.914 \\
\hline Alteplase dose, mean (SD) & $66.7(13.2)$ & $67.9(62.75-74.25)$ & $66.4(13.5)$ & 0.454 \\
\hline \multicolumn{5}{|l|}{ Stroke subtype $(\%)$} \\
\hline Atherothrombotic & $34 / 393(8.7)$ & $4 / 92(4.3)$ & $30 / 301(10.0)$ & 0.093 \\
\hline Cardioembolic & $130 / 393(33.1)$ & $32 / 92(34.8)$ & 98/301 (32.6) & 0.691 \\
\hline Lacunar & 45/393 (11.5) & $9 / 92(9.8)$ & $36 / 301(12.0)$ & 0.566 \\
\hline Large vessel, other & $39 / 393(9.9)$ & $7 / 92(7.6)$ & 32/301 (10.6) & 0.396 \\
\hline Multiple/unknown & $124 / 393(31.6)$ & $36 / 92(39.1)$ & $88 / 301(29.2)$ & 0.074 \\
\hline Unusual & $21 / 393(5.3)$ & $4 / 92(4.3)$ & $17 / 301(5.6)$ & 0.794 \\
\hline
\end{tabular}

$I Q R$, interquartile range; NIHSS, National Institutes of Health Stroke Scale; SD, standard deviation; TIA, transient ischemic attack 
Table 2 Radiological characteristics of the whole cohort of patients and of the two subgroups identified by presence/absence of CMBs

\begin{tabular}{|c|c|c|c|c|}
\hline & All $(n=434)$ & CMBs $(n=101)$ & No CMBs $(n=333)$ & $p$ value \\
\hline Early ischemic sign at baseline neuroimaging (\%) & $44 / 343(12.8)$ & $13 / 85(15.3)$ & $31 / 258(12.0)$ & 0.433 \\
\hline Hyperdense MCA sign (\%) & $53 / 341(15.5)$ & $8 / 84(9.5)$ & $45 / 257(17.5)$ & 0.079 \\
\hline \multicolumn{5}{|l|}{ CMB parameters } \\
\hline CMB number, mean (SD) & $0.57(2.02)$ & $2.45(3,61)$ & - & - \\
\hline $0 \mathrm{CMB}(n, \%)$ & $333 / 434(76.4)$ & - & & \\
\hline $1 \mathrm{CMB}(n, \%)$ & $60 / 434(13.8)$ & $60 / 101(59.4)$ & & \\
\hline $2-4 \mathrm{CMB}(n, \%)$ & $31 / 434(7.1)$ & $31 / 101(30.7)$ & & \\
\hline$\geq 5 \mathrm{CMB}(n, \%)$ & $10 / 434(2.3)$ & $10 / 101(9.9)$ & & \\
\hline CMB localization & - & & - & - \\
\hline Lobar (\%) & $74 / 434(17.1)$ & $74 / 101(73.3)$ & & \\
\hline Deep and infratentorial (\%) & $42 / 434(9.7)$ & 42/101 (41.6) & & \\
\hline Both lobar and deep/infratentorial (\%) & $15 / 434(3.5)$ & 15/101 (14.9) & & \\
\hline \multicolumn{5}{|l|}{ Fazekas WMD rating scale } \\
\hline $0(\%)$ & $140 / 434(32.3)$ & 18/101 (17.8) & $122 / 333(36.6)$ & $<0.001$ \\
\hline $1(\%)$ & $183 / 434(42.2)$ & $37 / 101(36.6)$ & $146 / 333(43.8)$ & 0.199 \\
\hline $2(\%)$ & $87 / 434(20.0)$ & $32 / 101(31.7)$ & $55 / 333(16.5)$ & 0.001 \\
\hline $3(\%)$ & $24 / 434(5.5)$ & 14/101 (13.9) & $10 / 333(3.0)$ & $<0.001$ \\
\hline \multicolumn{5}{|l|}{ Stroke territory } \\
\hline Anterior circulation $(\%)$ & $288 / 423(68.1)$ & $70 / 97(72.2)$ & $218 / 326(66.9)$ & 0.326 \\
\hline Posterior circulation $(\%)$ & $84 / 423$ (19.9) & $17 / 97(17.5)$ & $67 / 326(20.6)$ & 0.512 \\
\hline Both anterior and posterior (\%) & $51 / 423(12.1)$ & 10/97 (10.3) & 41/326 (12.6) & 0.547 \\
\hline \multicolumn{5}{|l|}{ Stroke localization } \\
\hline Cortical $(\%)$ & $141 / 421(33.5)$ & $25 / 97(25.8)$ & $116 / 324(35.8)$ & 0.066 \\
\hline Subcortical (\%) & $132 / 421(31.4)$ & 29/97 (29.9) & $103 / 324(31.8)$ & 0.724 \\
\hline Cortical-subcortical (\%) & $148 / 421(35.2)$ & $43 / 97(44.3)$ & $105 / 324(32.4)$ & 0.031 \\
\hline $\mathrm{ICH}(\mathrm{All})(\%)$ & $107 / 432(24.8)$ & $35 / 101(34.7)$ & 72/331 (21.8) & 0.009 \\
\hline $\mathrm{HI}(\%)$ & $52 / 432(12.0)$ & 12/101 (11.9) & 40/331 (12.1) & 0.956 \\
\hline $\mathrm{PH}(\%)$ & 49/432 (11.3) & 19/101 (18.8) & $30 / 331(9.1)$ & 0.007 \\
\hline PHr* $(\%)$ & $6 / 432(1.4)$ & 4/101 (4.0) & 2/331 (0.6) & 0.029 \\
\hline
\end{tabular}

*Of the 6 PHr, 4 had also PH and 2 also HI

$C M B$, cerebral microbleeds; $H I$, hemorrhagic infarction; $I C H$, intracerebral hemorrhage; $M C A$, middle cerebral artery; NIHSS, National Institutes of Health Stroke Scale; $P H$, parenchymal hemorrhage; $P H r$, remote parenchymal hemorrhage; $S D$, standard deviation, $W M D$, white matter disease

with CMBs (34.7\%) than in those without CMBs $(21.8 \%)(p=$ 0.009 ), particularly severe ICH (PH: $18.8 \%$ vs $9.1 \%, p=$ 0.007 ; PHr: $4.0 \%$ vs $0.6 \%, p=0.029$ ) (Table 2). HT resulted in symptomatic as per the SITS-MOST definition in $2(0.5 \%)$ patients (with both $\mathrm{PH}$ and $\mathrm{PHr}$ ) (Table 3). SICH as per any definition was more likely to develop in patients with CMBs (SICH/SITS-MOST, $2.0 \%$ vs $0, p=0.054$; SICH/NINDS, $12.9 \%$ vs $6.2 \%, p=0.041$; SICH/ECASS, $8.3 \%$ vs $2.2 \%, p$ $=0.015)($ Table 3$)$.

Mortality, both at 7 days and 90 days, did not significantly differ between patients with or without CMBs. Conversely, patients with $\mathrm{CMBs}$ were found to have a worse functional outcome (mRS 3-6) at 90 days more frequently compared with patients without CMBs $(42.9 \%$ vs $30.6 \%, p=0.031)$. Figure 1 reports the distribution of $\mathrm{mRS}$ scores in patients without and with CMBs.
Compared with those without any type of $\mathrm{ICH}$, the mean number of CMBs was significantly higher in patients with any ICH (0.98 vs $0.44, p=0.006)$, PH (0.69 vs $0.56, p=0.008)$, PHr (8.33 vs $0.46, p=0.003)$, and SICH by any definition (SICH/SITS-MOST: 11.0 vs $0.52, p=0.001$; SICH/NINDS: 1.39 vs $0.52, p=0.022$; SICH/ECASS: 2.46 vs $0.52, p=$ 0.004). A higher burden of CMBs was found more frequently in patients with any ICH (CMBs $2-4: 13.1 \%$ vs $5.2 \%, p=$ 0.006), $\mathrm{PH}$ (at least $1 \mathrm{CMB}: 22.4 \%$ vs $12.0 \%, p=0.042$; with a trend toward the statistical significance for CMBs 2-4: $14.3 \%$ vs $6.3 \%, p=0.069), \mathrm{PHr}(\mathrm{CMBs} \geq 5: 33.3 \%[2 / 6$ subjects] vs $1.9 \%$ [8/426], $p=0.007), \mathrm{SICH} / \mathrm{SITS}-\mathrm{MOST}$ (CMBs 2-4: $50 \%$ [1/2 subjects] vs 7.0\% [30/430], $p=$ 0.019; CMBs $\geq 5: 50 \%$ [1/2 subjects] vs $2.1 \%$ [9/430], $p=$ 0.046 ), SICH/NINDS (CMBs 2-4: $17.9 \%$ vs $6.3 \%, p=0.040$ ), and SICH/ECASS (CMBs $\geq 5: 15.4 \%$ vs $1.7 \%, p=0.030)$. 
Table 3 Outcome measures of the whole cohort of patients and of the two subgroups identified by the presence/absence of CMBs

\begin{tabular}{lllll}
\hline & All $(n=434)$ & CMBs $(n=101)$ & No CMBs $(n=333)$ & $p$ value \\
\hline Death at 7 days (\%) & $9 / 352(2.6)$ & $2 / 67(3.0)$ & $7 / 285(2.5)$ & 0.682 \\
mRS 3-6 at 3 months (\%) & $130 / 388(33.5)$ & $39 / 91(42.9)$ & $91 / 297(30.6)$ & 0.031 \\
Death at 3 months (\%) & $33 / 389(8.5)$ & $11 / 92(12.0)$ & $22 / 297(7.4)$ & 0.171 \\
SICH/SITS-MOST (\%) & $2 / 432(0.5 \%)$ & $2 / 101(2.0)$ & 0 & 0.054 \\
SICH/NINDS (\%) & $28 / 361(7.8)$ & $11 / 85(12.9)$ & $17 / 276(6.2)$ & 0.041 \\
SICH/ECASS (\%) & $13 / 360(3.6)$ & $7 / 84(8.3)$ & $6 / 276(2.2)$ & 0.015 \\
\hline
\end{tabular}

$m R S$, modified Rankin scale, SICH, symptomatic intracranial hemorrhage
Furthermore, the mean number of CMBs was higher in patients with unfavorable functional outcome ( 0.72 vs 0.53 , $p=0.040$ ) and at least $1 \mathrm{CMB}$ was found more frequently in these patients compared with those who reached a favorable outcome $(19.2 \%$ vs $10.1 \%, p=0.012)$.

Deep/infratentorial location of CMBs resulted significantly associated with any ICH $(16.8 \%$ vs $7.4 \%$ in patients without $\mathrm{ICH}, p=0.004)$, $\mathrm{PH}(20.4 \%$ vs $8.4 \%, p=0.017)$, while lobar CMBs were more frequently found in patients with $\mathrm{PHr}$ (66.7\% [4/6 subjects] vs $16.4 \%$ [70/426], $p=0.009$ ), $\mathrm{SICH} /$ SITS-MOST (100.0\% [2/2 subjects] vs $16.7 \%$ [72/430], $p=$ $0.029)$, and SICH/ECASS (46.2\% vs $16.4 \%, p=0.014)$, with only a trend toward the statistical significance for $\mathrm{SICH} /$ NINDS $(32.1 \%$ vs $16.5 \%, p=0.066)$.

Conversely, CMBs location seems not to have any correlation with mortality or functional outcome.

Regarding WMD, a higher burden of this radiological marker of cerebral SVD was significantly associated only with more severe ICH, i.e., PHr (Fazekas Rating Scale score 3: 33.3\% [2/6 subjects] vs 5.2\% [22/426], $p=0.039$ ) (but not with $\mathrm{SICH}$ ) and with mRS 3-6 at 90 days (Fazekas Rating Scale score 2: 26.2\% vs $17.1 \%$ in patients with 90 -day $\mathrm{mRS} 0-2, p=0.035$ ).

Multivariable regression analyses for outcome measures are reported in Tables 4 and 5. As regards $\mathrm{PH}$, after adjustment for the index stroke anatomical location (subcortical, corticalsubcortical), presence of $\mathrm{CMBs}$, number of CMBs as categorical variable, location of CMBs (deep/infrantentorial vs lobar), and atherothrombotic stroke subtype, the presence of CMBs independently predicted PH (OR 2.724, 95\% CI $1.360-5.464, p=0.005$ ) (Table 4 , section B). When the number of CMBs was included as a continuous variable in the model, the deep/infratentorial location of CMBs (OR 3.121, 95\% CI 1.342-7.259, $p=0.008$ ) resulted in an independent predictor of $\mathrm{PH}$ (Table 4, section B). Given the small numbers, no multivariable analysis was performed for $\mathrm{PHr}$ and $\mathrm{SICH}$, except for SICH/NINDS definition which either the presence or the number of CMBs as well as their location or WMD did not result independently associated with (Table 4, section C).

No independent association between the presence, number, or location of CMBs or WMD and either intra-hospital or 90day mortality as well as a functional outcome at 90 days was observed (Table 5).

The results of multivariate analyses did not change overall after the addition of time interval variables (i.e., onset-totreatment time, door-to-needle time, and bolus-MRI time) to the models (see e-Tables in the supplemental material).

\section{Discussion}

The objective of this study was to evaluate whether the presence of radiological markers of cerebral SVD could be a
Fig. 1 Distribution of mRS score in patients without and with CMBs

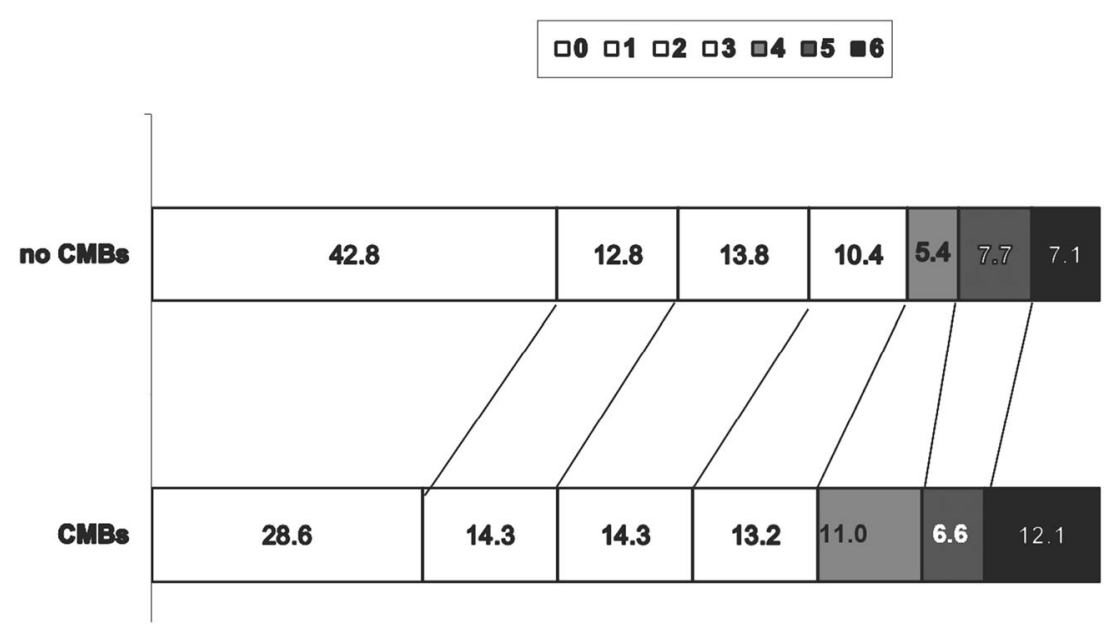


Table 4 Multivariable analyses for radiological outcome measures (HI, $\mathrm{PH}, \mathrm{SICH} / \mathrm{NINDS}$ )

\begin{tabular}{llll}
\hline & OR & $95 \%$ CI & $p$ \\
\hline A. Multivariable analysis for HI* & & & \\
Cortical-subcortical stroke & 2.957 & $1.508-5.797$ & 0.002 \\
Both anterior and posterior stroke & 4.142 & $1.804-9.509$ & 0.001 \\
Cardioembolic stroke & 2.337 & $1.229-4.443$ & 0.010 \\
WMD (absence) & 2.299 & $1.196-4.422$ & 0.013 \\
B. Multivariable analyses for PH & & & \\
First model** & & & \\
Cortical-subcortical stroke & 3.629 & $1.841-7.151$ & $<0.001$ \\
CMB (absence vs presence) & 2.724 & $1.360-5.464$ & 0.005 \\
Atherothrombotic stroke & 3.381 & $1.335-8.566$ & 0.010 \\
Second model*** & & & \\
Cortical-subcortical stroke & 3.804 & $1.934-7.481$ & $<0.001$ \\
Deep/infratentorial location of CMB & 3.121 & $1.342-7.259$ & 0.008 \\
Atherothrombotic stroke & 3.118 & $1.244-7.817$ & 0.015 \\
C. Multivariable analysis for SICH/NINDS**** & & \\
NIHSS at baseline & 1.119 & $1.050-1.193$ & 0.001 \\
Cortical-subcortical stroke & 3.123 & $1.265-7.708$ & 0.013 \\
\hline
\end{tabular}

*Adjusted for the history of diabetes mellitus, cortical-subcortical stroke, both anterior and posterior stroke, absence/presence of WMD at the modified Fazekas Rating Scale, cardioembolic stroke

**Adjusted for subcortical stroke, cortical-subcortical stroke, CMB location (deep/infratentorial vs lobar), CMB absence vs presence, CMB number as categorical variable, atherothrombotic stroke

***Adjusted for subcortical stroke, cortical-subcortical stroke, CMB location (deep/infratentorial vs lobar), CMB number as a continuous variable, atherothrombotic stroke

****Adjusted for NIHSS at baseline, cortical-subcortical stroke, hyperdense MCA sign, presence of CMBs, number of CMBs

possible limitation to the use of IVT. We found that although the presence of CMBs and their deep/infratentorial location independently predicted the development of large hemorrhagic transformation $(\mathrm{PH})$, they were not related either to SICH or unfavorable functional outcome.

In the last years, many research papers have been conducted on this topic. The prevalence of CMBs observed in our study cohort $(23.3 \%)$ was in keeping with that reported in the literature (range 15 to $38 \%$ ) $[12,13]$. Recently, some meta-analyses focusing on clinical outcomes have been also published.

A metanalysis [14], including 9 studies comprising 2479 patients with AIS receiving IVT, found that high CMB burden (>10 CMBs) on pre-treatment MRI was independently associated with SICH. These observations suggest that CMB burden may be included in individual risk stratification predicting the risk of SICH after treatment.

More recently, in a multicenter study analyzing 672 patients [15], the authors concluded that there is an increased risk of SICH after IVT when more than $10 \mathrm{CMBs}$ are present. Chacon-Portillo et al. [16] obtained similar results as regards
Table 5 Multivariable analyses for clinical outcome measures

\begin{tabular}{llll}
\hline & OR & $95 \%$ CI & $p$ \\
\hline $\begin{array}{l}\text { A. Death at 7 days* } \\
\text { NIHSS at baseline }\end{array}$ & 1.155 & $1.033-1.291$ & 0.012 \\
$\begin{array}{l}\text { B. mRS 3-6 at 3 months** } \\
\text { NIHSS at baseline }\end{array}$ & 1.302 & $1.185-1.431$ & $<0.001$ \\
Diabetes & 4.680 & $1.487-14.726$ & 0.008 \\
Cortical-subcortical stroke & 3.684 & $1.247-10.880$ & 0.018 \\
C. Death at 3 months*** & & & \\
NIHSS at baseline & 1.121 & $1.052-1.196$ & $<0.001$ \\
Age & 1.091 & $1.039-1.146$ & 0.001 \\
Diabetes & 3.080 & $1.223-7.755$ & 0.017 \\
Atherothrombotic stroke & 4.978 & $1.629-15.212$ & 0.005 \\
\hline
\end{tabular}

*Adjusted for Age, NIHSS at baseline, atherothrombotic stroke subtype **Adjusted for age, history of diabetes, past smoking, NIHSS at baseline, alteplase dose, blood glucose levels at baseline, atherothrombotic and lacunar stroke subtypes, cortical and cortical-subcortical strokes, posterior stroke, moderate WMD (modified Fazekas Scale equals to 2), presence/absence of CMBs, CMB number

***Adjusted for age, NIHSS at baseline, history of diabetes, history of $\mathrm{AF}$, atherothrombotic stroke subtype, cortical-subcortical stroke

CMB burden while they did not find any association between CMB presence or location with SICH.

Similarly to our study, the last recently published large meta-analysis [13] reported that patients with CMBs had increased risk of $\mathrm{PH}$ and $\mathrm{PHr}$ but not of SICH. Concerning outcome measures, five or more and $>10 \mathrm{CMBs}$ independently predicted poor outcome; conversely, in our cohort, either the presence or the burden of CMBs as well as their location did not result in independent predictors of 90-day mortality and unfavorable functional outcome in adjusted analyses.

In all these studies, CMB burden is the parameter that seems to be strongly associated with the risk of $\mathrm{ICH}$; of note, the number of patients with high CMB burden (CMBs > 10) was very low. Results regarding clinical outcome are conflicting.

In our study, despite the fact that patients with CMBs and, among these, those with higher mean number of CMBs were more likely to develop any type of ICH (except for the milder one, i.e., HI), whether symptomatic or not, it was evident that presence, burden, or location of CMBs did not have any impact on either intra-hospital or 90-day mortality as well as on functional outcome at 90 days. Similarly, despite the significant univariate association between the higher burden of WMD and severe ICH (i.e., PH) and 90-day unfavorable outcome, WMD did not result in an independent predictor of $\mathrm{SICH}$, mortality, or functional outcome in adjusted analyses. Therefore, in our cohort, patients with moderate to severe leukoaraiosis appeared to still have clinical benefit from IV rt-PA, although an increased risk of symptomatic ICH in these patients is described [4]. NIHSS at baseline is the parameter that strongly correlates with clinical outcome in our study. 
As regards the location of CMBs, in our study, their position in deep/infratentorial brain areas resulted in an independent predictor of PH. Data analysis showed that CMBs were lobar in the majority of our patients. In the literature, it is reported that more than $87 \%$ of CMBs located in the lobar regions and predominantly in the posterior cortex, are likely caused by cerebral amyloid angiopathy (CAA). On the other hand, hypertensive microbleeds predominantly develop in the deep gray matter of the brainstem [17]. No multivariable analysis was performed for $\mathrm{PHr}$, due to the small numbers, but most patients with PHr had CMBs and, in particular, lobar CMBs.

Our study has limitations. First, given the retrospective design and the objectives of this study, patients were not consecutively included because only patients who underwent MRI within approximately $24 \mathrm{~h}$ were taken into consideration. However, the percentage of patients (around 15\%) excluded from the analysis is relatively small and overall acceptable. Second, only few patients underwent MR before thrombolysis, and hence, for CMB and WMD assessment, we used the follow-up MRI which, based on our local protocol, is usually performed within $24 \mathrm{~h}$ from symptoms onset. Although studies are still limited, a possible appearance of new CMBs in the first $24 \mathrm{~h}$ after thrombolysis has been described [18]. However, even hypothesizing a post-IVT increase of CMBs in our series of patients, this would have increased rather than reduced the possible relationship between $\mathrm{CMBs}$ and $\mathrm{SICH}$ or unfavorable outcomes.

Because of the small number of pre-IVT MRI in our patient cohort and since we did not systematically collect data on the exact location of hemorrhagic transformation, we were not able to evaluate, for those patients who developed a local hemorrhagic transformation of the index infarct and/or a remote ICH after IVT, whether there was a relationship between the specific location of $\mathrm{PH}$ and $\mathrm{PHr}$ and previous presence of CMBs or WMD in the same site. This can be considered likely particularly for CMBs, also based on available data from populationbased studies which found a spatial colocalization between the site of CMB and the region of hemorrhagic stroke [19].

However, these aspects should be thoroughly investigated in large prospective studies on ischemic stroke patients receiving acute reperfusion/revascularization treatments.

Another potential limitation of our study, similarly to all studies on CMBs, is that patients undergoing a good-quality MRI (in contrast to computed tomography) are usually less severely ill and more collaborative, which may represent a potential selection bias.

Finally, the small number of SICHs lowers statistical power. Assessment of the association of the individual number of CMBs with bleeding risk was complicated by the low rate of patients with a large number of CMBs (CMBs > 10). Therefore, in our cohort conclusions, concerning the rare patients with a great burden of CMBs cannot be drawn. For this reason, further analyses in larger studies taking into account CMBs number and location, and their relationship with measures of functional outcome, are needed.

Current evidence points out that patients with CMBs should not be ruled out from a potential beneficial therapy as IVT. In the absence of comparative data demonstrating lack of functional benefit from IVT in AIS patients with CMBs vs those without, all available data do not justify the exclusion of eligible candidates to administration of IV rt-PA on the basis of CMB presence in case MRI, besides CT scan or alone, is performed in the acute setting.

Acknowledgments Open access funding provided by Università degli Studi di Roma La Sapienza within the CRUI-CARE Agreement. Special thanks to Professor D. Toni for his assistance and guidance in this research.

Authors' contributions MLC and DT researched literature and conceived the study. All authors were involved in protocol development and patient recruitment. SL was involved in data analysis. MLC and SL wrote the first draft of the manuscript. All authors reviewed and edited the manuscript and approved the final version of the manuscript

Data availability Not applicable.

\section{Compliance with ethical standards}

Conflict of interest The authors declare that they have no conflict of interest

Ethical approval This research study was conducted retrospectively from data obtained for clinical purposes. All of the procedures were performed in accordance with the Declaration of Helsinki.

Consent to participate Not applicable.

Consent for publication Not applicable.

Open Access This article is licensed under a Creative Commons Attribution 4.0 International License, which permits use, sharing, adaptation, distribution and reproduction in any medium or format, as long as you give appropriate credit to the original author(s) and the source, provide a link to the Creative Commons licence, and indicate if changes were made. The images or other third party material in this article are included in the article's Creative Commons licence, unless indicated otherwise in a credit line to the material. If material is not included in the article's Creative Commons licence and your intended use is not permitted by statutory regulation or exceeds the permitted use, you will need to obtain permission directly from the copyright holder. To view a copy of this licence, visit http://creativecommons.org/licenses/by/4.0/.

\section{References}

1. Viswanathan A, Chabriat H (2006) Cerebral microhemorrhage. Stroke 37:550-555

2. Greenberg SM, Vernooij MW, Cordonnier C, Viswanathan A, alShahi Salman R, Warach S, Launer LJ, van Buchem M, Breteler 
MM, Microbleed Study Group (2009) Cerebral microbleeds: a guide to detection and interpretation. Lancet Neurol 8:165-174

3. Pantoni L, Fierini F, Poggesi A (2014) Thrombolysis in acute stroke patients with cerebral small vessel disease. Cerebrovasc Dis 37:513

4. Demchuk AM, Khan F, Hill MD, Barber PA (2008) et NINDS rtPA Stroke Study Group. Importance of leukoaraiosis on CT for tissue plasminogen activator decision making: evaluation of the NINDS rt-PA stroke study. Cerebrovasc Dis 26:120-125

5. Toni D, Mangiafico S, Agostoni E, Bergui M, Cerrato P, Ciccone A, Vallone S, Zini A, Inzitari D (2015) Intravenous thrombolysis and intra-arterial interventions in acute ischemic stroke: Italian Stroke Organisation (ISO)-SPREAD guidelines. Int J Stroke 10(7):1119-1129

6. Cordonnier C, Potter GM, Jackson CA, Doubal F, Keir S, Sudlow CLM, Wardlaw JM, Salman RAS (2009) Observer MicroBleed Scale (BOMBS) Improving interrater agreement about brain microbleeds: development of the brain. Stroke 40:94-99

7. Fazekas F, Chawluk JB, Alavi A, Hurtig HI, Zimmerman RA (1987) MR signal abnormalities at $1.5 \mathrm{~T}$ in Alzheimer's dementia and normal aging. AJR Am J Roentgenol 149:351-356

8. Larrue V, von Kummer R, Muller A et al (2001) Risk factors for severe hemorrhagic transformation in ischemic stroke patients treated with recombinant tissue plasminogen activator: a secondary analysis of the European-Australasian Acute Stroke Study (ECASS II). Stroke 32:438-441

9. Wahlgren N, Ahmed N, Dávalos A, Ford GA, Grond M, Hacke W, Hennerici MG, Kaste M, Kuelkens S, Larrue V, Lees KR, Roine RO, Soinne L, Toni D, Vanhooren G (2007) SITS MOST investigators. Thrombolysis with alteplase for acute ischaemic stroke in the safe implementation of thrombolysis in stroke-monitoring study (SITS-MOST): an observational study. Lancet 369:275-282

10. The National Institute of Neurological Disorders and Stroke rt-PA Stroke Study Group (1995) Tissue plasminogen activator for acute ischemic stroke. N Engl J Med 333:1581-1587

11. van Swieten J, Koudstaal P, Visser M et al (1988) Interobserver agreement for the assessment of handicap in stroke patients. Stroke 19:604-607

12. Yates PA, Villemagne VL, Ellis KA et al (2014) Cerebral microbleeds: a review of clinical, genetic, and neuroimaging associations. Front Neurol 4:205
13. Charidimou A, Turc G, Oppenheim C, Yan S, Scheitz JF, Erdur H, Klinger-Gratz PP, el-Koussy M, Takahashi W, Moriya Y, Wilson D, Kidwell CS, Saver JL, Sallem A, Moulin S, Edjlali-Goujon M, Thijs V, Fox Z, Shoamanesh A, Albers GW, Mattle HP, Benavente OR, Jäger HR, Ambler G, Aoki J, Baron JC, Kimura K, Kakuda W, Takizawa S, Jung S, Nolte CH, Lou M, Cordonnier C, Werring DJ (2017) Microbleeds, cerebral hemorrhage, and functional outcome after stroke thrombolysis: individual patient data meta-analysis. Stroke 48:2084-2090

14. Tsivgoulis G, Zand R, Katsanos AH, Turc G, Nolte CH, Jung S, Cordonnier C, Fiebach JB, Scheitz JF, Klinger-Gratz PP, Oppenheim C, Goyal N, Safouris A, Mattle HP, Alexandrov AW, Schellinger PD, Alexandrov AV (2016) Risk of symptomatic intracerebral hemorrhage after intravenous thrombolysis in patients with acute ischemic stroke and high cerebral microbleed burden. A Meta-analysis JAMA Neurol 73(6):675-683

15. Zand R, Tsivgoulis G, Singh M, McCormack M, Goyal N, Ishfaq MF, Shahripour RB, Nearing K, Elijovich L, Alexandrov AW, Liebeskind DS, Alexandrov AV (2017) Cerebral microbleeds and risk of intracerebral hemorrhage post intravenous thrombolysis. J Stroke Cerebrovasc Dis 26(3):538-544

16. Chacon-Portillo MA, Llinas RH, Marsh EB (2018) Cerebral microbleeds shouldn't dictate treatment of acute stroke: a retrospective cohort study evaluating risk of intracerebral hemorrhage. BMC Neurol 18:33

17. Juyoun L, Eun Hee S, Eungseok O et al (2018) Characteristics of cerebral microbleeds. Dement Neurocogn Disord 17(3):73-82

18. Kimura K, Aoki J, Shibazaki K, Saji N, Uemura J, Sakamoto Y (2013) New appearance of extraischemic microbleeds on T2*weighted magnetic resonance imaging 24 hours after tissue-type plasminogen activator administration. Stroke 44:2776-2781

19. Akoudad S, Portegies MLP, Koudstaal PJ, Hofman A, van der Lugt A, Ikram MA, Vernooij MW (2015) Cerebral microbleeds are associated with an increased risk of stroke. The Rotterdam Study Circulation 132(6):509-516

Publisher's note Springer Nature remains neutral with regard to jurisdictional claims in published maps and institutional affiliations. 